\title{
Measurement of Brain Edema by Noninvasive Cerebral Electrical Impedance in Patients with Massive Hemispheric Cerebral Infarction
}

\author{
Jin He Lou ${ }^{a}$ Jian Wang ${ }^{a}$ Li Xu Liu ${ }^{b}$ Lan Ying He ${ }^{b}$ Hao Yang ${ }^{c}$ Wei Wei Dong ${ }^{b}$ \\ a Department of Neurology, The Second Affiliated Hospital, and ${ }^{\mathrm{b}}$ Department of Neurology, \\ The First Affiliated Hospital, Chongqing Medical University, and ${ }^{\mathrm{C} C o l l e g e ~ o f ~ E l e c t r i c a l ~ E n g i n e e r i n g, ~}$ \\ Chongqing University, Chongqing, China
}

\section{Key Words}

Brain edema - Massive hemispheric cerebral infarction •

Cerebral electric impedance

\begin{abstract}
Background: Malignant cerebral infarction often occurs in patients with massive cerebral infarction. Monitoring brain edema is therefore helpful to make correct clinical decisions. Our previous studies have confirmed that cerebral electrical impedance (CEI) can sensitively reflect the brain edema after stroke. Methods: The CEI was measured consecutively by a noninvasive brain edema monitor in 69 patients with massive hemispheric cerebral infarction (MHCl). The results of the CEl were converted into the perturbation index (PI). The characteristics of dynamic changes of the $\mathrm{CEI}$ after $\mathrm{MHCl}$ were analyzed. Receiver-operating characteristics analysis was used to calculate predictive values for $\mathrm{PI}$ and other known parameters including NIHSS score and infarct volume. Results: (1) The overall rate of positive CEI was $88.4 \%$ $(61 / 69)$ in all patients with $\mathrm{MHCl}$. (2) The PI on the infarct side increased significantly within $24 \mathrm{~h}$ after stroke onset and reached a peak level $3-5$ days after stroke onset $(p<0.01)$. (3) Age, NIHSS score at admission, infarct volume, and the PI at
\end{abstract}

$24 \mathrm{~h}$ after stroke onset were significantly different between the malignant and nonmalignant groups. The best predictor of a malignant $\mathrm{MHCl}$ was the $\mathrm{Pl}$ at $24 \mathrm{~h}$ after stroke onset with a cut-off value of 10.02 (90.9\% sensitivity, $87.2 \%$ specificity, $76.9 \%$ positive predictive value, $95.3 \%$ negative predictive value). Conclusions: The noninvasive CEI can sensitively reflect the brain edema in patients with $\mathrm{MHCl}$. Monitoring the CEI may help to predict malignant $\mathrm{MHCl}$ and guide treatment decisions.

Copyright $\odot 2012$ S. Karger AG, Basel

\section{Introduction}

Malignant cerebral infarction accounts for up to 10$20 \%$ of all massive hemispheric cerebral infarctions (MHCI) and is associated with a high mortality estimated at $80 \%$ with standard conservative treatment [1-3]. $\mathrm{MHCI}$ is most common in middle cerebral artery (MCA) infarctions and the clinical deterioration occurs 1-7 days after stroke onset.

Active conservative treatment may be ineffective in patients with extensive ischemic brain edema after MHCI. Several studies have demonstrated that decompressive

\section{KARGER}

Fax +41613061234 E-Mail karger@karger.ch www.karger.com
(C) 2012 S. Karger AG, Basel

$0014-3022 / 12 / 0686-0350 \$ 38.00 / 0$

Accessible online at:

www.karger.com/ene
Prof. Jian Wang

Department of Neurology, The Second Affiliated Hospita

Chongqing Medical University, Linjiangmen 74

Chongqing 400010 (China)

E-Mail jian.wx@163.com 
hemicraniectomy can reduce the brain edema and intracranial pressure, and therefore improve prognosis [4-6]. If it can be done accurately, dynamically measuring the brain edema in patients with MHCI is useful to predict malignant infarctions at bedside and select the correct treatment timely. The cerebral electrical impedance (CEI) is a physical parameter reflecting the migration of brain tissue water and the change in total brain water [7-10]. In our previous studies, we have found that the CEI can be measured by a noninvasive method and its safety has been confirmed. Noninvasive CEI measurement is useful for monitoring brain edemas and hematomas [11-14]. The present study used noninvasive CEI measurements to detect brain edemas in patients with MHCI. In addition, we assessed the accuracy of the CEI in predicting malignant MHCI and compared the CEI with other known predictive values for a malignant course.

\section{Materials and Methods}

\section{Patient Data}

In this prospective pilot study, we evaluated 69 patients with MHCI consecutively admitted to the Department of Neurology at the First Affiliated Hospital of Chongqing Medical University between July 2003 and July 2009, including 31 patients with large artery atherosclerosis (LAA) and 38 patients with cerebral embolism (CE) according to the Trial of Org 10172 in Acute Stroke Treatment (TOAST) criteria. The inclusion criteria which had to be met were the following: (1) acute ischemic infarction involving the MCA territory; (2) time of stroke onset established precisely; (3) National Institutes of Health Stroke Scale (NIHSS) score at admission; (4) MRA or CT angiogram showing occlusion of the intracranial carotid artery or MCA main stem; (5) native CT was performed at admission and follow-up CT or MRI examinations were performed within $24 \mathrm{~h}$ after stroke onset if the initial CT examination showed no visible abnormality, and (6) the patients had no other organic brain disorders.

All patients were admitted to the critical care unit and were treated according to standardized protocols. They were treated by osmotherapy and mechanically ventilated if necessary. None of the patients received thrombolytic therapy or required endovascular intervention. The clinical signs for malignant MHCI were checked at least every $2 \mathrm{~h}$ by an experienced neurologist. Malignant MHCI was defined as anisocoria or death attributable to herniation. Hemicraniectomy was considered in the event of rapid neurological deterioration and deterioration in the level of consciousness, or when the patient developed clinical signs of uncal herniation. The protocol was approved by the Ethics Committee of the First Affiliated Hospital of Chongqing Medical University and informed consent was obtained from the patients' family members.

Baseline clinical information and demographic characteristics were extracted from the medical records, including NIHSS score at admission, time of symptom onset, vascular risk factors, stroke subtype and treatment.

\section{Method of CEI Measurement}

The method of CEI measurement has been described in our previous studies [11, 14]. Briefly, the CEI was measured using a low-frequency, bioelectrical, noninvasive brain edema monitor (Chongqing Born-Fruk Medical Equipment Co. Ltd, Chongqing, China). The skin was locally shaved and sterilized by acetone or 95\% alcohol. Three gel electrodes were placed: one at the left frontal, one at the right frontal and one in the middle of the occipital, respectively. An invariable current of $50 \mathrm{kHz}$ and $0.1 \mathrm{~mA}$ was given into a person's brain. The results of the CEI were converted into the perturbation index (PI). The CEI of all patients with MHCI was measured consecutively for at least 7 days. In addition, only the stable PI value obtained at least $3 \mathrm{~h}$ after osmotherapy by hyperosmolar agents could be used for data analysis.

\section{Imaging Protocol}

All CT examinations were performed using a 64-slice CT scanner (GE). The settings for the native CT were as follows: 120 $\mathrm{kV}$ tube voltage, $280 \mathrm{mAs}$ tube current, $6.0 \mathrm{~mm}$ slice reconstruction, $0.625 \mathrm{~mm}$ collimation, $0.516 \mathrm{~mm}$ pitch. Those for the CT angiogram were as follows: $120 \mathrm{kV}$ tube voltage, $600 \mathrm{mAs}$ tube current, $0.625 \mathrm{~mm}$ collimation, $0.516 \mathrm{~mm}$ pitch, $0.4 \mathrm{~s}$ rotation time, $4 \mathrm{~s}$ total scan time, $220 \mathrm{~mm}$ display field of view, $0.3 \mathrm{~mm}$ reconstruction interval. The scan range was from the aortic arch to the top of the head. $70 \mathrm{ml}$ iohexol, $370 \mathrm{mg} / \mathrm{ml}$ at $5 \mathrm{ml} / \mathrm{s}$, was administered via the antecubital vein with a Medrad pressure injector. The scan started automatically when the CT value was more than $180 \mathrm{HU}$.

All MRI examinations were performed with a 1.5-T MRI unit (GE Signa MR/i). The settings for the native MRI were as follows: $5 \mathrm{~mm}$ slice thickness, $1 \mathrm{~mm}$ layer interval. Those for the $\mathrm{T}_{1^{-}}$ weighted image (T1WI) were: TR $660 \mathrm{~ms}$, TE $11.6 \mathrm{~ms}$; $\mathrm{T}_{2}$-weighted image (T2WI): TSE sequence, TR 4,000 ms, TE $99 \mathrm{~ms}$. Those for the diffusion-weighted image (DWI) were: echo-planar pulse sequence, TR/TE 10,000/80, the diffusion-weighted gradient field was imposed on the $\mathrm{x}$-, $\mathrm{y}$ - and $\mathrm{z}$-axes simultaneously, $\mathrm{b}=2,000$, $128 \times 128$ matrix, single excitation, $40 \mathrm{~s}$ scan time. And those for the MRA were: three-dimensional time-of-flight magnetic resonance, FC, TR/TE 25/6.9, FA: 20 degrees, 240-350 mm field of view, $512 \times 256$ matrix, $1.0-1.2 \mathrm{~mm}$ slice thickness.

The volumes of the infarctions were calculated automatically by an image analyzing system (Institute of Image and Graphics, School of Computer Science and Engineering, Sichuan University, Chengdu, China) according to the cranial MRI or CT (scanned by a Uniscan D1000 high-definition scanner, Beijing, China).

\section{Statistical Analysis}

Spearman's correlation coefficient was used to analyze the correlation between the PI and the volume of the edema. $\mathrm{p}<0.05$ was considered statistically significant. Categorical variables were compared with a 2-tailed Fisher exact test, and quantitative variables were compared with a 2-tailed Student t test. We determined the discriminatory power and the optimal cut-off values for the differentiation between the patient groups using receiveroperating characteristic curves. Cut-off values were chosen to maximize the sum of sensitivity and specificity. Two-sided confidence intervals were calculated using the Wilson procedure with a correction for continuity. 
Fig. 1. Comparison between CT, MRI and the PI in a patient with left MHCI. A 49-year-old woman was measured $12 \mathrm{~h}$ after stroke onset, PI on the left $=10.11$, PI on the right $=8.66$. The native CT $12 \mathrm{~h}$ after stroke onset showed an infarct lesion with slightly low density (a). MRI-T2WI (c) and DWI (d) showed a massive high signal in the left hemisphere.
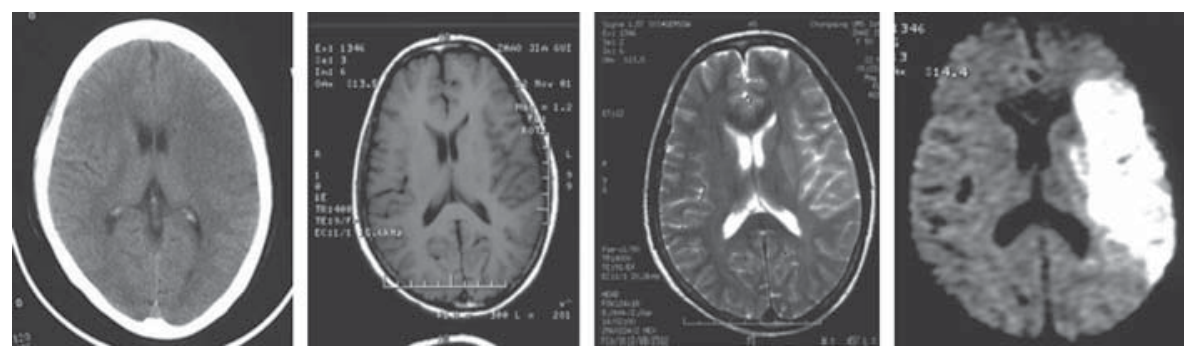

\section{Results}

\section{Infarct Volume and Positive CEI Rate}

The infarct volume of the 69 patients with MHCI was $169.7 \pm 60.0 \mathrm{ml}(79.4-315.2 \mathrm{ml})$. The infarct volumes in the patients with LAA and CE were $164.7 \pm 65.5 \mathrm{ml}$ and $175.7 \pm 51.9 \mathrm{ml}$, respectively. There was no significant difference between the two groups ( $\mathrm{p}=0.456$; table 1 ).

The range of the PI in all patients was between 6.74 and 13.16. The CEI was defined as positive when the difference of the PI between the infarct side and the contralateral side was $>0.3$, or when the PI on the infarct side was $>9.5$, which was in accordance with the CT or MRI scans. The overall rate of positive CEI was $88.4 \%$ (61/69). The rate of positive CEI was higher in the patients with LAA $(93.5 \%, 29 / 31)$ than in the patients with CE $(84.2 \%, 32 / 38)$, but the difference lacked statistical significance $(\mathrm{p}=$ 0.408 ; table 1).

\section{Dynamic Changes of the CEI}

After admission, the PI on the infarct side increased in $81.2 \%(56 / 69)$ of the patients. Figure 1 depicts a patient with left MHCI. The native CT $12 \mathrm{~h}$ after stroke onset showed an infarct lesion with slightly low density. The PI was higher on the infarct side than on the contralateral side (10.11 vs. 8.66). On the MRI scans, T2WI and DWI showed a large infarct focus in the left MCA territory.

The PI on the infarct side was higher than on the contralateral side at admission $(9.48 \pm 1.25$ vs. $8.71 \pm 1.47$; $\mathrm{n}=69 ; \mathrm{p}<0.05$ ). The difference in the PI between the infarct side and the contralateral side was more significant 3 days after stroke onset $(10.27 \pm 1.41$ vs. $8.96 \pm 1.83$; $\mathrm{n}=67 ; \mathrm{p}<0.01)$, and 5 days after stroke onset (10.39 \pm 1.95 vs. $8.89 \pm 1.69 ; \mathrm{n}=57 ; \mathrm{p}<0.01)$. The PI gradually decreased 7 days after stroke onset, but it was still higher on the infarct side than on the contralateral side (10.07 \pm 1.72 vs. $8.74 \pm 1.54 ; \mathrm{n}=50 ; \mathrm{p}<0.05$; table 2 ).

Dynamic measurement results showed that the PI on the infarct side did not increase in $11.6 \%$ (8/69) of the pa-
Table 1. Positive CEI rate in the patients with MHCI

\begin{tabular}{lllll}
\hline $\begin{array}{l}\text { Type of } \\
\text { MHCI }\end{array}$ & Patients & $\begin{array}{l}\text { Infarct } \\
\text { volume, } m l\end{array}$ & $\begin{array}{l}\text { Number of } \\
\text { positive CEI }\end{array}$ & $\begin{array}{l}\text { Rate of pos- } \\
\text { itive CEI, \% }\end{array}$ \\
\hline LAA & 31 & $164.7 \pm 65.5$ & 29 & 93.5 \\
CE & 38 & $175.7 \pm 51.9^{*}$ & 32 & $84.2^{* *}$ \\
\hline Total & 69 & $169.7 \pm 60.0$ & 61 & 88.4 \\
\hline
\end{tabular}

Compared with LAA: ${ }^{*} \mathrm{p}=0.620,{ }^{* *} \mathrm{p}=0.408$.

Table 2. Change of the CEI value at different points in time in the patients with MHCI

\begin{tabular}{|c|c|c|c|c|}
\hline \multirow{2}{*}{$\begin{array}{l}\text { Time of } \\
\text { measurement }\end{array}$} & \multirow{2}{*}{$\begin{array}{l}\mathrm{Pa}- \\
\text { tients }\end{array}$} & \multicolumn{2}{|l|}{ CEI } & \multirow[t]{2}{*}{$\mathrm{p}$} \\
\hline & & $\begin{array}{l}\text { infarct } \\
\text { side }\end{array}$ & $\begin{array}{l}\text { contra- } \\
\text { lateral side }\end{array}$ & \\
\hline At admission & 69 & $9.48 \pm 1.25$ & $8.71 \pm 1.47$ & $<0.05$ \\
\hline 3 days after onset & 67 & $10.27 \pm 1.41$ & $8.96 \pm 1.83$ & $<0.01$ \\
\hline 5 days after onset & 57 & $10.39 \pm 1.95$ & $8.89 \pm 1.69$ & $<0.01$ \\
\hline 7 days after onset & 52 & $10.07 \pm 1.72$ & $8.74 \pm 1.54$ & $<0.05$ \\
\hline
\end{tabular}

tients, this figure was even lower than on the contralateral side for at least 7 days after stroke onset: among the $11.6 \%$ were $6.5 \%(2 / 31)$ of the patients with LAA and $15.8 \%(6 / 38)$ patients with CE. The average infarct volume of these patients was $147.5 \mathrm{ml}(79-189 \mathrm{ml})$. None of these patients developed malignant MHCI.

Figure 2 is from a 69 -year-old woman with massive CE who was hospitalized due to a sudden weakness in her right limb with dysarthria for $3 \mathrm{~h}$. She had a history of rheumatic heart disease and atrial fibrillation (AF). MRI on the 3rd day after stroke onset showed a massive infarct focus in the left MCA territory, but without severe brain edema. The PI on the left and right side was 8.14 and 9.20, respectively, $18 \mathrm{~h}$ after stroke onset. The PI on the infarct 


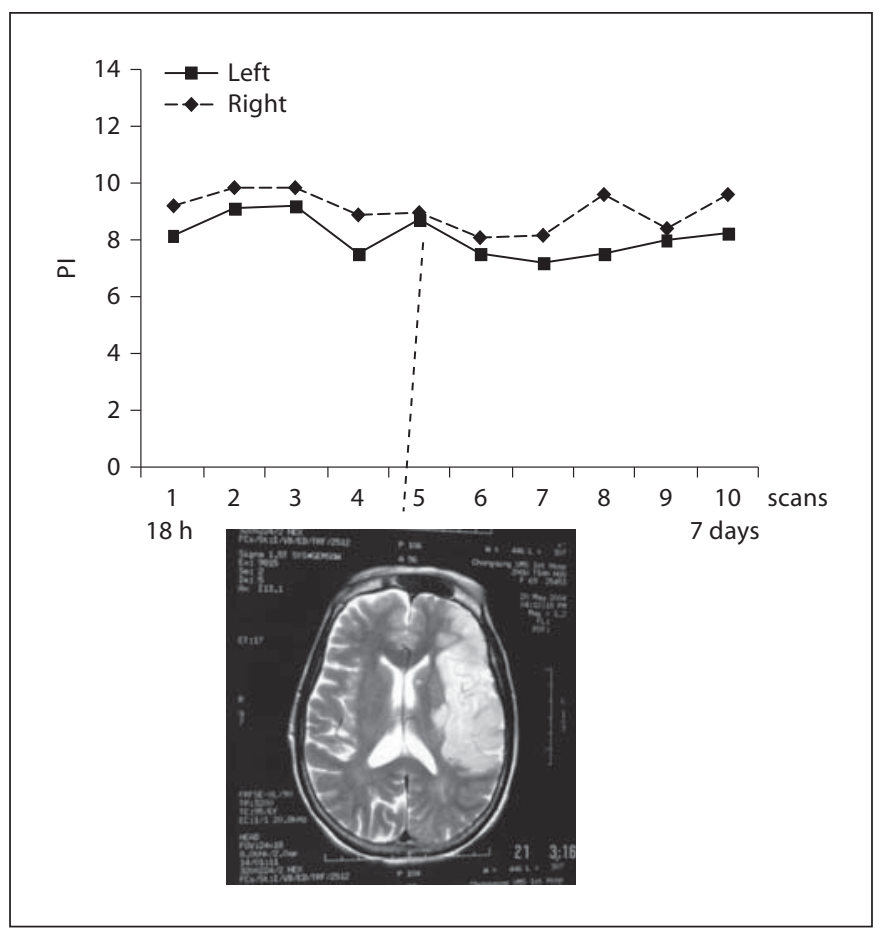

Fig. 2. Dynamic changes of the PI in a patient with left massive CE. MRI on the 3rd day after stroke onset showed a massive infarct focus in the left MCA territory, but no severe brain edema. The PI on the infarct side did not increase significantly and remained lower than on the contralateral side for at least 7 days.

side did not increase significantly and remained lower than on the contralateral side during the following week.

Figure 3 is from a 72-year-old man with massive CE who was hospitalized due to a sudden hemiplegia on the left side and aphasia for $10 \mathrm{~h}$. He had a history of AF. MRI on the 3rd day after stroke onset showed a massive infarct focus in the right MCA territory; however, the midline shifted only slightly to the left. The PI on the left and right side was 9.0 and 7.12, respectively, $12 \mathrm{~h}$ after stroke onset. The PI on the infarct side did not increase and remained lower than on the contralateral side during the following week.

\section{Correlation between the PI and Infarct Volume}

There was a positive correlation between the PI on the infarct side $24 \mathrm{~h}$ after stroke onset and the infarct volume $(\mathrm{n}=69 ; \mathrm{r}=0.684 ; \mathrm{p}<0.001$; fig. 4$)$.

\section{Comparison between the Malignant and the}

Nonmalignant Group

The main characteristics of the patients are summarized in table 3 . A total of 22 patients (31.9\%) developed a

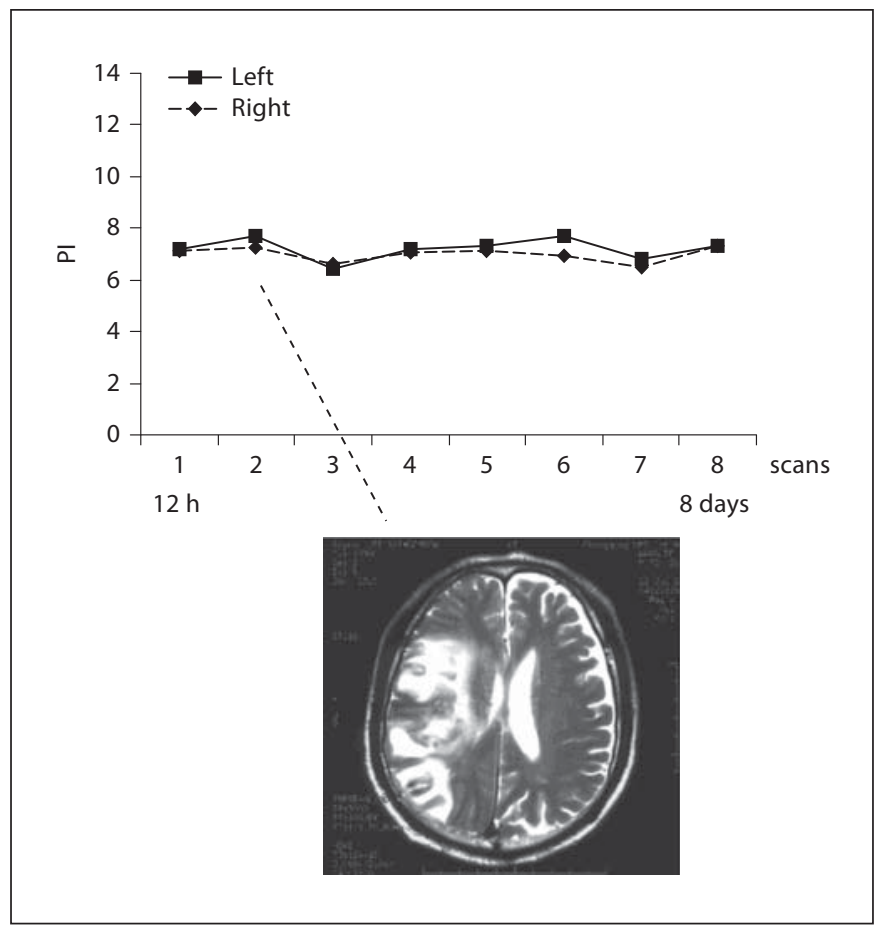

Fig. 3. Dynamic changes of the PI in a patient with right massive CE. MRI on the 3rd day after stroke onset showed a massive infarct focus in the right MCA territory and the midline shifted slightly to the left. The PI on the infarct side did not increase and remained lower than on the contralateral side for at least 7 days.

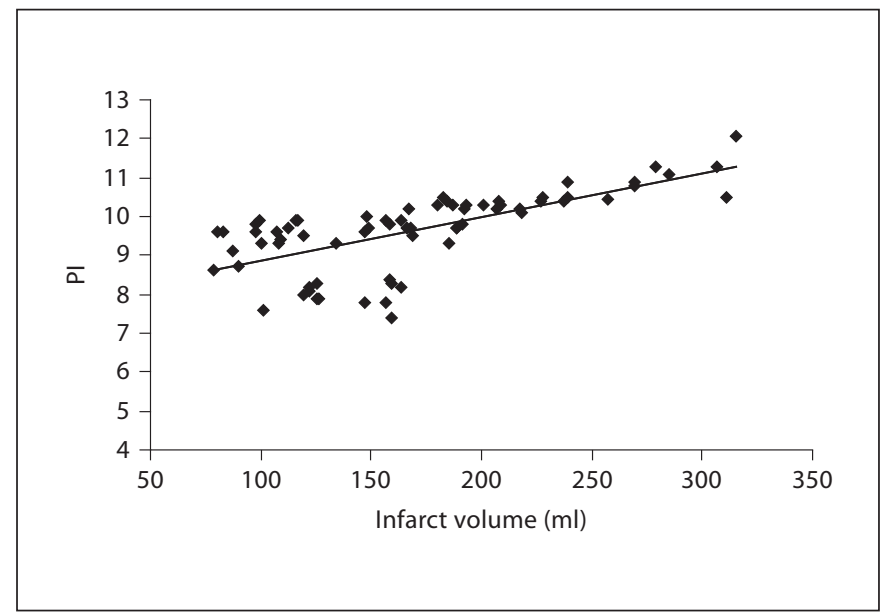

Fig. 4. Correlation between the PI on the infarct side at $24 \mathrm{~h}$ after stroke onset and the infarct volume $(n=69 ; \mathrm{r}=0.684 ; \mathrm{p}<0.001)$. 
Table 3. Baseline demographic and clinical characteristics

\begin{tabular}{|c|c|c|c|}
\hline Patient characteristics & $\begin{array}{l}\text { Malignant } \\
\text { MHCI }(n=22)\end{array}$ & $\begin{array}{l}\text { Nonmalignant } \\
\text { MHCI }(\mathrm{n}=47)\end{array}$ & $\mathrm{p}$ \\
\hline Mean age $\pm S D$, years & $56.7 \pm 6.4$ & $70.4 \pm 9.4$ & $<0.001$ \\
\hline Male & $14(63.6 \%)$ & $28(59.6 \%)$ & 0.747 \\
\hline Left MCA infarction & $10(45.4 \%)$ & $23(48.9 \%)$ & 0.787 \\
\hline \multicolumn{4}{|l|}{ Vascular risk factors } \\
\hline History of hypertension & $15(68.2 \%)$ & $34(72.3 \%)$ & 0.723 \\
\hline History of diabetes & $4(18.2 \%)$ & $11(23.4 \%)$ & 0.860 \\
\hline Atrial fibrillation & $13(59.1 \%)$ & $25(53.2 \%)$ & 0.646 \\
\hline Coronary heart disease & $7(31.8 \%)$ & $17(36.2 \%)$ & 0.724 \\
\hline Smoking & $8(36.4 \%)$ & $14(29.8 \%)$ & 0.585 \\
\hline Ischemic stroke & $2(9.1 \%)$ & $6(12.8 \%)$ & 0.967 \\
\hline \multicolumn{4}{|l|}{ Stroke subtype (TOAST classification) } \\
\hline Atherothrombotic & $10(45.5 \%)$ & $28(59.6 \%)$ & 0.272 \\
\hline Cardioembolic & $11(50.0 \%)$ & $17(36.2 \%)$ & 0.276 \\
\hline Median NIHSS score at admission (quartiles) & $22.4(20,25)$ & $15.9(13,19)$ & $<0.001$ \\
\hline Mean infarct volume $\pm \mathrm{SD}, \mathrm{ml}$ & $234.8 \pm 46.1$ & $139.6 \pm 38.0$ & $<0.001$ \\
\hline Mean PI at $24 \mathrm{~h} \pm \mathrm{SD}$ & $10.52 \pm 0.58$ & $9.24 \pm 0.86$ & $<0.001$ \\
\hline \multicolumn{4}{|l|}{ Mean vital signs and laboratory values \pm SD } \\
\hline Systolic blood pressure, $\mathrm{mm} \mathrm{Hg}$ & $153.7 \pm 24.8$ & $148.7 \pm 21.1$ & 0.562 \\
\hline Diastolic blood pressure, $\mathrm{mm} \mathrm{Hg}$ & $86.4 \pm 11.7$ & $82.9 \pm 14.1$ & 0.465 \\
\hline Body temperature, ${ }^{\circ} \mathrm{C}$ & $37.4 \pm 0.5$ & $37.1 \pm 0.4$ & 0.247 \\
\hline Glucose levels, mg/dl & $155.3 \pm 46.7$ & $147.1 \pm 34.2$ & 0.642 \\
\hline White blood cell count, $\times 10^{6} / \mathrm{ml}$ & $8.9 \pm 2.3$ & $8.1 \pm 1.7$ & 0.523 \\
\hline
\end{tabular}

Table 4. Predictability of malignant MHCI

\begin{tabular}{lllll}
\hline Predicting factors & Sensitivity, $\%$ & Specificity, \% & $\begin{array}{l}\text { Positive predictive } \\
\text { value, \% }\end{array}$ & $\begin{array}{l}\text { Negative predictive } \\
\text { value, } \%\end{array}$ \\
\hline NIHSS score at admission $>19$ & $81.8(59.7-94.7)$ & $78.7(64.3-89.3)$ & $64.3(43.8-81.1)$ & $90.2(76.6-97.1)$ \\
Infarct volume $>187$ ml & $72.7(49.8-89.2)$ & $80.9(66.7-90.8)$ & $64.0(42.5-82.0)$ & $86.4(72.6-94.8$ \\
PI at 24 h after stroke onset $>10.02$ & $90.9(70.8-98.6)$ & $87.2(74.2-95.1)$ & $76.9(56.7-91.3)$ & $95.3(84.2-99.3)$ \\
\hline
\end{tabular}

Values in parentheses are $95 \%$ confidence intervals.

malignant MHCI. 18 of these patients died from brain hernia; 2 of them had undergone hemicraniectomy. Among the 18 patients who had died, 12 died between 2 and 5 days after stroke onset, the other 6 died between 5 and 7 days after stroke onset. The remaining 4 patients who had developed brain hernia survived; all of them had hemicraniectomy performed 20-90 h after stroke onset (mean: $42 \mathrm{~h}$ ) because of the development of clinical signs of brain hernia.

As shown in table 3, the patients with malignant MHCI were significantly younger and had higher NIHSS scores at admission than those with nonmalignant MHCI. The infarct volume was greater in the malignant group than in the nonmalignant group. Moreover, the PI value at $24 \mathrm{~h}$ after stroke onset in the malignant group was higher than in the nonmalignant group.

\section{Discriminant Analysis}

Using the receiver-operating characteristic curves analysis, we calculated the cut-off values of clinical measures and imaging examination-based volume measurements with the highest predictive values for the development of malignant MHCI (table 4). Compared with the NIHSS score at admission and the infarct volume, the PI 
at $24 \mathrm{~h}$ after stroke onset had a relatively high predictive value $(90.9 \%$ sensitivity, $87.2 \%$ specificity, $76.9 \%$ positive predictive value, $95.3 \%$ negative predictive value).

\section{Discussion}

We measured the CEI dynamically in patients with MHCI by a noninvasive method. The results showed that the PI increased significantly within $24 \mathrm{~h}$ after stroke onset in most patients and reached a peak level 3-5 days after stroke onset. Moreover, we identified that the PI at $24 \mathrm{~h}$ after stroke onset could help to predict the development of malignant MHCI.

Compared with the results of our previous study, the overall positive rate of the PI was higher in patients with MHCI (88.4\%) than in patients with an infarct volume $<20 \mathrm{ml}(42.9 \%)$ and between 20 and $50 \mathrm{ml}$ (75.9\%) [14]. In addition, we found that the PI on the infarct side at admission increased significantly in more than $80 \%$ of the patients. The results indicated that a noninvasive CEI measurement could be more sensitive to reflect an early (within $24 \mathrm{~h}$ after stroke onset) brain edema in patients with MHCI. This is reasonable because patients with MHCI have more serious brain edemas. The mean PI at admission was higher on the infarct side than on the contralateral side $(\mathrm{p}<0.05)$ due to severe brain edemas at this early stage, which suggested that the patient might suffer from MHCI if the PI increased significantly within $24 \mathrm{~h}$ after stroke onset, even if the native CT did not show significant low-density lesions at this time. Dynamic CEI measurement results showed that the PI on the infarct side continued to increase after admission and reached a peak level 3-5 days after stroke onset. It tended to decrease 7 days after stroke onset, but it was still higher than on the contralateral side $(\mathrm{p}<0.05)$. The dynamic changes of the PI in patients with MHCI were consistent with the evolution of ischemic brain edemas; however, the PI in patients with MHCI increased more significantly due to more severe brain edemas [14].

Surprisingly, we found that the PI on the infarct side did not increase in $6.5 \%(2 / 31)$ of the patients with LAA and $15.8 \%(6 / 38)$ of the patients with CE. The dynamic measurement showed that this phenomenon sustained for at least 7 days. The reason is unclear. It is well known that brain edemas can cause brain swelling, which implies a new constituent is added to the extracellular space of the brain. Excluding a tumor, the new constituent can only come from blood vessels, therefore brain swelling after cerebral infarction can be caused only by both isch- emia and reperfusion [15]. When the intracranial large artery or its main trunk is blocked by an embolus from the extracranial artery, the blood flow may be completely rather than partly interrupted. If the embolus cannot be dissolved and it is too late to establish an effective collateral circulation due to acute arterial embolism, the infarct region will have no blood supply. In that case the brain water content will not change and there will be no significant brain tissue swelling, although brain cells will die. The CEI per se is a physical parameter reflecting the brain water content, therefore it is reasonable that the CEI will not change significantly if the brain water content does not change. Moreover, when the cerebral blood flow is interrupted completely, nerve cells will die rapidly by autophagy due to ischemia and hypoxia, accompanied with cell lysis and electrolyte overflow, which can increase the conductivity of the brain tissue rate, therefore the PI on the infarct side will decline, even be lower than on the contralateral side. This phenomenon also suggests that not all patients with MHCI have severe brain edemas, therefore individual therapy should be given. At present, there is a lack of consensus regarding the application of osmotherapy against brain edema after cerebral infarction, including when, how much and how long hyperosmolar agents such as mannitol should be used. Dynamic CEI measurements might help to guide osmotherapy in patients with MHCI.

Brain edemas after ischemic stroke usually reach their peak between 3 and 5 days after stroke. However, in a given patient it is not predictable at which time the edema causes significant brain tissue shifts with subsequent herniation and clinical deterioration [16]. Several parameters were identified to allow a prediction of the malignant cerebral infarction, including clinical factors such as younger age, a history of hypertension or heart failure, a high NIHSS score, coma at admission, anisocoria, early nausea and vomiting, as well as imaging predictors such as a CT showing a low-density range $>50 \%$ of the MCA territory and a DWI volume $>145 \mathrm{ml}$ [17-21]. But so far, there was no predictor with enough sensitivity for clinical decision-making. In this study, we compared the malignant and nonmalignant group and identified several parameters that were associated with a malignant course, including younger age, a higher NIHSS score at admission, a larger infarct volume, and a higher PI at $24 \mathrm{~h}$ after stroke onset. We chose the PI at $24 \mathrm{~h}$ after stroke onset as a predictor of brain hernia because the CEI is a dynamic parameter after stroke. Receiver-operating characteristic curves analysis of all parameters identified the PI at $24 \mathrm{~h}$ after stroke onset with a cut-off value of $>10.02$ as the 
most powerful predictor for the development of a malignant infarction. This is reasonable because that CEI reflects a brain edema more directly than the infarct volume and NIHSS score. The brain edema after ischemic stroke represents an additional volume within the fixed internal volume of the skull. Whether brain hernia occurs depends on the degree of severity of the brain edema. As shown in this study, although there is a positive correlation between the PI on the infarct side and the infarct volume, a large cerebral infarction does not necessarily develop into a severe brain edema. Therefore, monitoring cerebral edemas by CEI might be more helpful in predicting malignant infarction than the NIHSS score and imaging examination. In addition, an advantage of the CEI is that it can be monitored conveniently and real-time CEI changes can be obtained at the bedside.

The PI at $24 \mathrm{~h}$ after stroke onset achieved only moderate sensitivity (90.9\%) and specificity (87.2\%), although both are higher than for the NIHSS score and infarct volume. We speculated that one of the reasons was that our study included a high proportion of elderly patients aged $>70$. Because a certain degree of age-related cerebral atrophy protects from space-occupying brain swelling, those elderly patients with severe cerebral edemas do not necessarily develop brain herniation. Secondly, the CEI per se has individual differences which may have some impact on its predictive values. Although the positive predictive value of the CEI is not very high (76.9\%), a PI $>10.02$ at $24 \mathrm{~h}$ after stroke onset suggests a great risk of malignant infarction; however, a PI $>10.02$ at $24 \mathrm{~h}$ after stroke onset suggests a small risk of malignant infarction because of the high negative predictive value of the CEI (95.3\%).

There are some limitations to this study. (1) We could not exclude the effect of cerebral hemorrhagic conversion on the CEI value for a lack of follow-up CT or MRI. (2) We could not affirm that the occlusive artery had recanalized or that the infarct tissue received reperfusion for a lack of follow-up MRA or CT angiograms, which additionally would be helpful in interpreting why the PI of some patients did not increase for a week. (3) Similar to previous studies, the information obtained on impedance is limited by the few electrodes used in this study. Multiple electrodes and electrical impedance imaging may help to solve this problem.

In conclusion, this study has shown that the noninvasive CEI can sensitively reflect the degree of severity of brain edemas after MHCI. The noninvasive CEI monitoring may help to predict the likelihood of a malignant cerebral infarction in patients with MHCI and to make correct clinical decisions. Therefore it is worthy of further studies.

\section{Acknowledgements}

This study was supported by a grant from the National Natural Science Foundation Key Project (basic research of bioelectrical impedance image monitoring technology, No. 50337020).

\section{References}

1 Henderson GV: Management of massive cerebral infarct. Curr Neurol Neurosci Rep 2004;4:497-504.

-2 Berrouschot J, Sterker M, Bettin S, Köster J, Schneider D: Mortality of space-occupying ('malignant') middle cerebral artery infarction under conservative intensive care. Intensive Care Med 1998;24:620-623.

- 3 Hacke W, Schwab S, Horn M, Spranger M, De Georgia M, von Kummer R: 'Malignant' middle cerebral artery territory infarction: clinical course and prognostic signs. Arch Neurol 1996;53:309-315.

4 Köhrmann M, Schwab S: Hemicraniectomy for malignant middle cerebral artery infarction. Curr Opin Crit Care 2009;15:125-130.

5 Vahedi K: Decompressive hemicraniectomy for malignant hemispheric infarction. Curr Treat Options Neurol 2009;11:113-119.
-6 Swiat M, Targosz-Gajniak M, Arkuszewski M, Wojtacha M, Bazowski P, Opala G, Pieta $\mathrm{M}$, Gamrot J: Decompressive hemicraniectomy in ischaemic stroke. Neurol Neurochir Pol 2010;44:131-138.

7 Holder DS: Detection of cerebral ischaemia in the anaesthetised rat by impedance measurement with scalp electrodes: implications for noninvasive imaging of stroke by electrical impedance tomography. Clin Phys Physiol Meas 1992;13:63-75.

-8 Schuier FJ, Hossmann KA: Experimental brain infarcts in cats. II. Ischemic brain edema. Stroke 1980;11:593-601.

-9 Suga S, Mitani S, Shimamoto Y, Kawase T, Toya S, Sakamoto K, Kanai H, Fukui M, Takeneka N: In vivo measurement of intra- and extracellular space of brain tissue by electrical impedance method. Acta Neurochir 1990;51:22-24.
10 Lingwood BE, Dunster KR, Colditz PB, Ward LC: Noninvasive measurement of cerebral bioimpedance for detection of cerebral edema in the neonatal piglet. Brain Res 2002; 945:97-105.

11 Liu L, Dong W, Ji X, Chen L, Chen L, He W, Jia J: A new method of noninvasive brainedema monitoring in stroke: cerebral electric impedance measurement. Neurol Res 2006;28:31-37.

12 Liu LX, Dong WW, Wang J, Wu Q, He W, Jia YJ: The role of noninvasive monitoring of cerebral impedance in stroke. Acta Neurochir Suppl 2005;95:137-140.

13 Liu LX, Dong WW, Jia JP, Wang J, Chen LH: Application of noninvasive cerebral electrical impedance measurement on brain edema in patients with intracerebral hemorrhage. Chinese J Neurol 2007;40:383-386. 
- 14 He LY, Wang J, Luo Y, Dong WW, Liu LX: Application of noninvasive cerebral electrical impedance measurement on brain edema in patients with cerebral infarction. Neurol Res 2010;32:770-774

- 15 Simard JM, Kent TA, Chen M, Tarasov KV, Gerzanich V: Brain oedema in focal ischaemia: molecular pathophysiology and theoretical implications. Lancet Neurol 2007;6: 258-268.

16 Shaw CM, Alvord EC Jr, Berry RG: Swelling of the brain following ischemic infarction with arterial occlusion. Arch Neurol 1959;1: 161-177.
17 Treadwell SD, Thanvi B: Malignant middle cerebral artery (MCA) infarction: pathophysiology, diagnosis and management. Postgrad Med J 2010;86:235-242.

-18 Thomalla GJ, Kucinski T, Schoder V, Fiehler J, Knab R, Zeumer H, Weiller C, Röther J: Prediction of malignant middle cerebral artery infarction by early perfusion- and diffusion-weighted magnetic resonance imaging. Stroke 2003;34:1892-1899.

-19 Kasner SE, Demchuk AM, Berrouschot J, Schmutzhard E, Harms L, Verro P, Chalela JA, Abbur R, McGrade H, Christou I, Krieger DW: Predictors of fatal brain edema in massive hemispheric ischemic stroke. Stroke 2001;32:2117-2123.
20 Oppenheim C, Samson Y, Manaï R, Lalam T, Vandamme X, Crozier S, Srour A, Cornu P, Dormont D, Rancurel G, Marsault C: Prediction of malignant middle cerebral artery infarction by diffusion-weighted imaging. Stroke 2000;31:2175-2181.

-21 Arenillas JF, Rovira A, Molina CA, Grivé E, Montaner J, Alvarez-Sabín J: Prediction of early neurological deterioration using diffusion- and perfusion-weighted imaging in hyperacute middle cerebral artery ischemic stroke. Stroke 2002;33:2197-2203. 